Supplement of Hydrol. Earth Syst. Sci., 22, 547-565, 2018

https://doi.org/10.5194/hess-22-547-2018-supplement

(C) Author(s) 2018. This work is distributed under

the Creative Commons Attribution 3.0 License.

(c) (i)

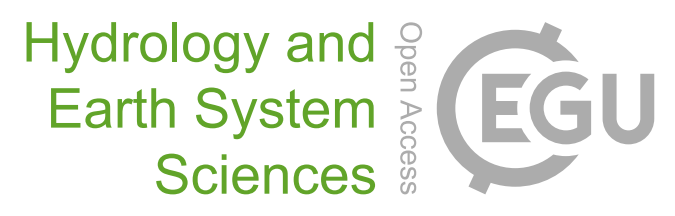

Supplement of

\title{
Quantifying human impacts on hydrological drought using a combined modelling approach in a tropical river basin in central Vietnam
}

\begin{abstract}
A. B. M. Firoz et al.
\end{abstract}
Correspondence to: A. B. M. Firoz (abm.firoz@th-koeln.de)

The copyright of individual parts of the supplement might differ from the CC BY 3.0 License. 


\begin{tabular}{lcc}
\hline \multicolumn{2}{c}{ Flow of the Vu Gia $\left(\mathrm{m}^{3} \mathrm{~s}-1\right)$} & $\begin{array}{c}\text { Diversion } \\
\text { Rate (\%) }\end{array}$ \\
\cline { 1 - 2 } Before Quang Hue & After Quang Hue & 34.6 \\
90.0 & 65.4 & 35.2 \\
80.0 & 58.3 & 36.2 \\
70.0 & 51.0 & 36.6 \\
60.0 & 44.4 & 37.7 \\
50.0 & 37.4 & 38.4 \\
40.0 & 30.8 & 39.2 \\
30.0 & 24.3 & 40.3 \\
20.0 & 17.9 & 43.0
\end{tabular}

Table S1. The water diversion rules at the Quang Hue river from Vu Gia to Thu Bon. Before Quang Hue represent the actual flow of Vu Gia before the diversion. After Quang Hue, represent the remaining water that is continuing to flow to the Vu Gia 5 through the Ai Nghia station. The Diversion Rate (\%) represents the amount which diverted from the Vu Gia to Thu Bon river and flows over Giao Thuy Station. 
(a) A Vuong (MOIT, 2011a)

\begin{tabular}{|l|r|r|r|r|r|}
\hline Month & Flood Control & Upper Zone & Normal Zone & Lower Zone & Dead Level \\
\hline Jan & 380 & 380 & 380 & 376 & 340 \\
\hline Feb & 380 & 380 & 375 & 376 & 340 \\
\hline Mar & 380 & 378 & 372 & 374.1 & 340 \\
\hline Apr & 380 & 374.2 & 370 & 371 & 340 \\
\hline May & 380 & 369.5 & 361 & 366 & 340 \\
\hline Jun & 380 & 365.7 & 352 & 361.1 & 340 \\
\hline Jul & 380 & 362.3 & 349 & 354.7 & 340 \\
\hline Aug & 380 & 357.2 & 342 & 347.4 & 340 \\
\hline Sep & 380 & 352.2 & 342 & 340 & 340 \\
\hline Oct & 380 & 353 & 349 & 350 & 340 \\
\hline Nov & 380 & 369 & 369 & 364 & 340 \\
\hline Dec & 380 & 378 & 373 & 364 & 340 \\
\hline
\end{tabular}

(b) Dak Mi 4 (MOIT, 2011b)

\begin{tabular}{|l|r|r|r|r|}
\hline Month & Flood Control & Upper Zone & Normal Zone & Dead Level \\
\hline Jan & 258 & 258 & 255 & 240 \\
\hline Feb & 258 & 258 & 256 & 240 \\
\hline Mar & 258 & 258 & 255 & 240 \\
\hline Apr & 258 & 256 & 253 & 240 \\
\hline May & 258 & 252.7 & 249 & 240 \\
\hline Jun & 258 & 251 & 248 & 240 \\
\hline Jul & 258 & 249.7 & 246 & 240 \\
\hline Aug & 258 & 244 & 241 & 240 \\
\hline Sep & 258 & 240.3 & 240 & 240 \\
\hline Oct & 258 & 240 & 240 & 240 \\
\hline Nov & 258 & 244.5 & 240.2 & 240 \\
\hline Dec & 258 & 253 & 247 & 240 \\
\hline
\end{tabular}

(c) Song Bung 4 (MOIT, 2013)

\begin{tabular}{|l|r|r|r|r|}
\hline Month & Flood Control & Upper Zone & Normal Zone & Dead Level \\
\hline Jan & 222.6 & 222.5 & 216.9 & 205 \\
\hline Feb & 222.6 & 222.5 & 215.3 & 205 \\
\hline Mar & 222.6 & 222.5 & 212.4 & 205 \\
\hline
\end{tabular}




\begin{tabular}{|l|r|r|r|r|}
\hline Apr & 222.6 & 222.5 & 208.7 & 205 \\
\hline May & 222.6 & 222.5 & 206.9 & 205 \\
\hline Jun & 222.6 & 222.5 & 205.7 & 205 \\
\hline Jul & 222.6 & 219.4 & 205.3 & 205 \\
\hline Aug & 222.6 & 215.5 & 205 & 205 \\
\hline Sep & 222.6 & 218 & 205 & 205 \\
\hline Oct & 222.6 & 220 & 205 & 205 \\
\hline Nov & 222.6 & 222.5 & 213.8 & 205 \\
\hline Dec & 222.6 & 222.5 & 216.8 & 205 \\
\hline
\end{tabular}

(d) Song Bung 5 (MOIT, 2012b)

\begin{tabular}{|l|r|r|r|}
\hline Month & Flood Control & Normal Zone & Dead Level \\
\hline Jan & 60 & 60 & 58.5 \\
\hline Feb & 60 & 60 & 58.5 \\
\hline Mar & 60 & 60 & 58.5 \\
\hline Apr & 60 & 60 & 58.5 \\
\hline May & 60 & 60 & 58.5 \\
\hline Jun & 60 & 60 & 58.5 \\
\hline Jul & 60 & 60 & 58.5 \\
\hline Aug & 60 & 60 & 58.5 \\
\hline Sep & 60 & 60 & 58.5 \\
\hline Oct & 60 & 60 & 58.5 \\
\hline Nov & 60 & 60 & 58.5 \\
\hline Dec & 60 & 60 & 58.5 \\
\hline
\end{tabular}

(e) Song Bung 6 (MOIT, 2012a)

\begin{tabular}{|l|r|r|r|}
\hline Month & Flood Control & Normal Zone & Dead Level \\
\hline Jan & 34 & 34 & 31.8 \\
\hline Feb & 34 & 34 & 31.8 \\
\hline Mar & 34 & 34 & 31.8 \\
\hline Apr & 34 & 34 & 31.8 \\
\hline May & 34 & 34 & 31.8 \\
\hline Jun & 34 & 34 & 31.8 \\
\hline Jul & 34 & 34 & 31.8 \\
\hline Aug & 34 & 34 & 31.8 \\
\hline Sep & 34 & 34 & 31.8 \\
\hline Oct & 34 & 34 & 31.8 \\
\hline
\end{tabular}




\begin{tabular}{|l|l|l|l|}
\hline Nov & 34 & 34 & 31.8 \\
\hline Dec & 34 & 34 & 31.8 \\
\hline
\end{tabular}

(f) Song Tranh 2 (MOIT, 2012c)

\begin{tabular}{|l|r|r|r|}
\hline Month & Flood Control & Normal Zone & Dead Level \\
\hline Jan & 175 & 174.24 & 138 \\
\hline Feb & 175 & 173.85 & 138 \\
\hline Mar & 175 & 168.48 & 138 \\
\hline Apr & 175 & 155.31 & 138 \\
\hline May & 175 & 153.33 & 138 \\
\hline Jun & 175 & 140.82 & 138 \\
\hline Jul & 175 & 139.51 & 138 \\
\hline Aug & 175 & 142.44 & 138 \\
\hline Sep & 175 & 150.44 & 138 \\
\hline Oct & 175 & 165.92 & 138 \\
\hline Nov & 175 & 174.31 & 138 \\
\hline Dec & 175 & 174.73 & 138 \\
\hline
\end{tabular}

(g) Song Con (MOIT, 2008)

\begin{tabular}{|l|r|r|r|r|}
\hline Month & Flood Control & Upper Zone & Normal Zone & Dead Level \\
\hline Jan & 340 & 340 & 334.52 & 319 \\
\hline Feb & 340 & 340 & 331.76 & 319 \\
\hline Mar & 340 & 337.45 & 328.45 & 319 \\
\hline Apr & 340 & 335.42 & 322.86 & 319 \\
\hline May & 340 & 334.74 & 321.36 & 319 \\
\hline Jun & 340 & 330 & 320.37 & 319 \\
\hline Jul & 340 & 325.77 & 319.00 & 319 \\
\hline Aug & 340 & 319 & 319.00 & 319 \\
\hline Sep & 340 & 340 & 319.00 & 319 \\
\hline Oct & 340 & 340 & 319.40 & 319 \\
\hline Nov & 340 & 340 & 334.02 & 319 \\
\hline Dec & 340 & 340 & 336.17 & 319 \\
\hline
\end{tabular}

5

Table S2- Operation Rules of the hydropower operation of the VGTB; (a) A Vuong, (b) Dak Mi 4, (c) Song Bung 4, (d) Song Bung 5, (e) Song Bung 6, (f) Song Tranh 2, (g) Song Con 


\begin{tabular}{|l|l|l|l|}
\hline Calibrated parameters & Short description & $\begin{array}{l}\text { calibrated } \\
\text { Value }\end{array}$ & Range \\
\hline soilMaxDPS & Maximum depression storage capacity & 2 & $1.0-5$ \\
\hline soilMaxInfSummer & Maximum infiltration in summer & 40 & $1-200$ \\
\hline soilMaxInfWinter & Maximum infiltration in winter & 100 & $1-200$ \\
\hline soilDistMPSLPS & MPS/LPS distribution coefficient & 0.68 & $0-1$ \\
\hline soilDiffMPSLPS & MPS/LPS diffusion coefficient & 0.4 & $0-1$ \\
\hline soilConcRD1 & Recession coefficient for overland flow & 1.2 & $1.0-3.0$ \\
\hline soilConcRD2 & Recession coefficient for interflow & 3.5 & $2.0-10$ \\
\hline soilPolRed & Potential reduction coeffiecient for aET & 3 & $1.0-10$ \\
\hline soilMaxPerc & computation & & $1.0-20$ \\
\hline gwRG1Fact & Maximum percolation rate & 20 & $0.1-10$ \\
\hline gwRG2Fact & Adaptation of the fast groundwater outflow & 1 & $0.1-10$ \\
\hline gwRG1RG2dist & Adaptation of the baseflow & 0.4 & $0-1$ \\
\hline flowRouteTA & RG1-RG2 distribution coefficient & 0.5 & $1-100$ \\
\hline & River routing coefficient & 10 & \\
\hline
\end{tabular}

Table S3. Parameters selected for the model calibration (other parameters of the model were left to default values during 5 calibration) 

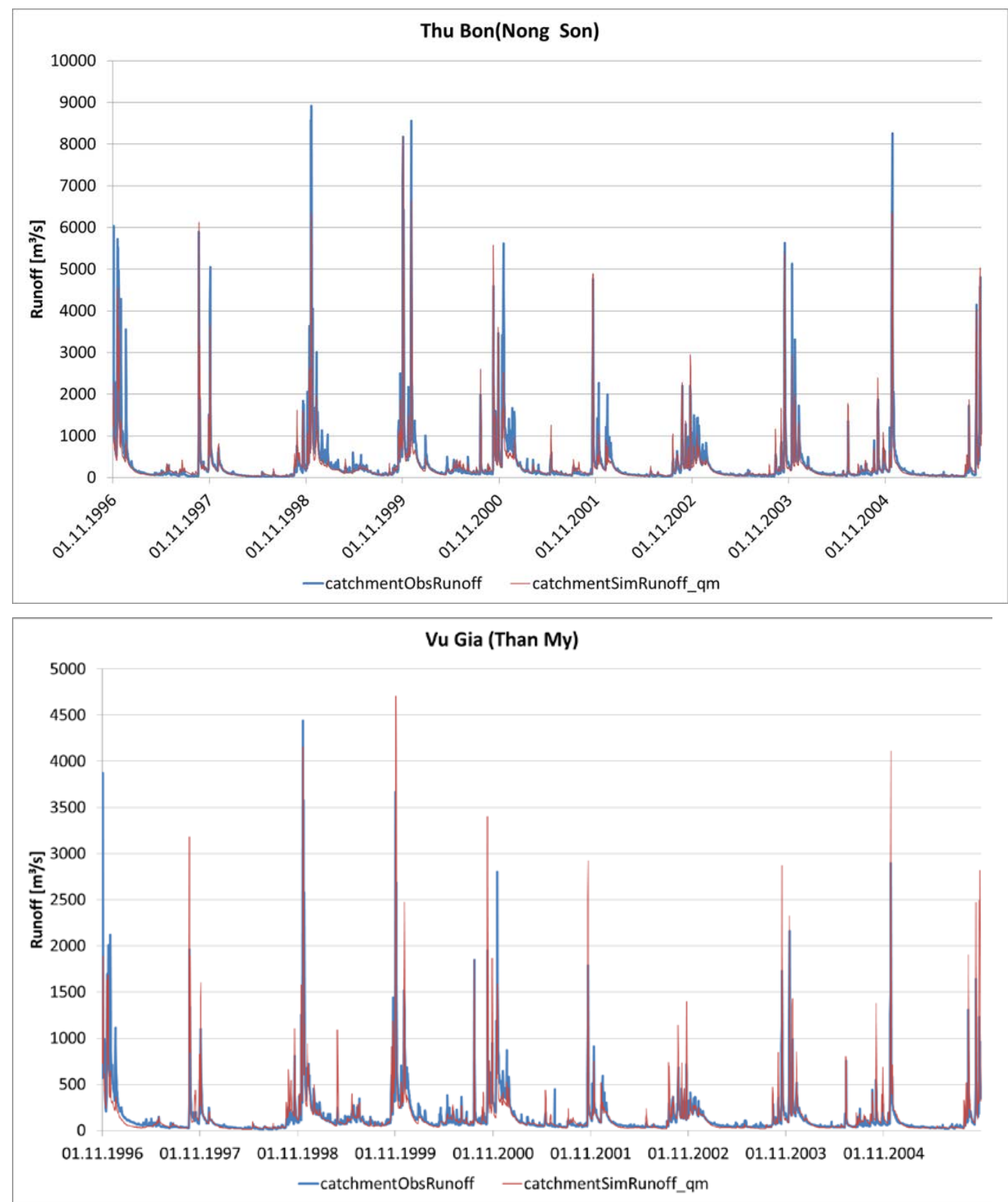

Figure S4. J2000 Hydrograph simulation compared to observed discharge (1996-2005) for the Thu Bon at Nong Son and Vu 5 Gia river at Thanh My station. 

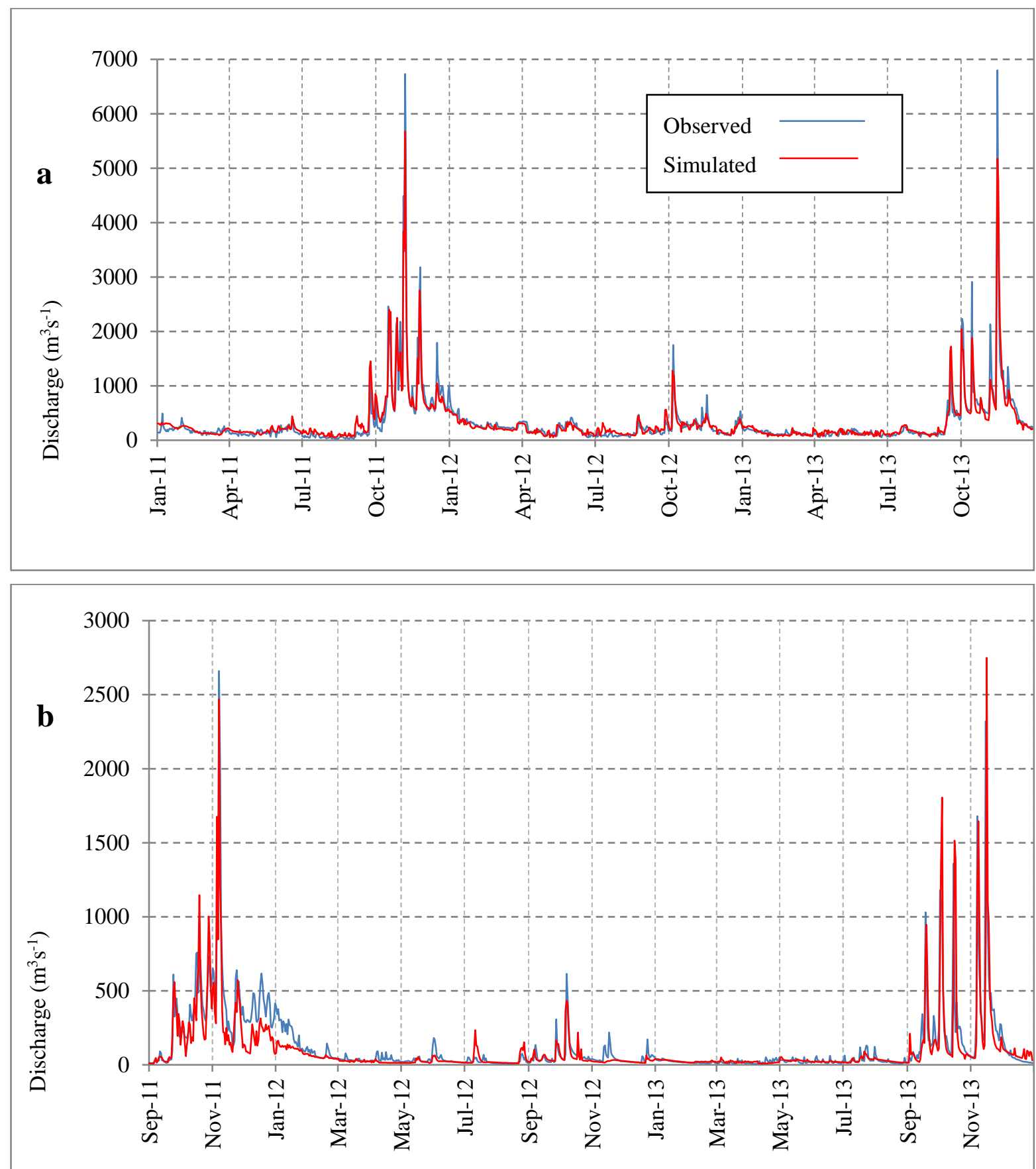

Figure- S5. Simulated reconstructed model output (Reservoir Simulation) compared to observed discharge from 2011 to 2013, for a) Thu Bon at Nong Son station, b) Vu Gia at Thanh My Station. 


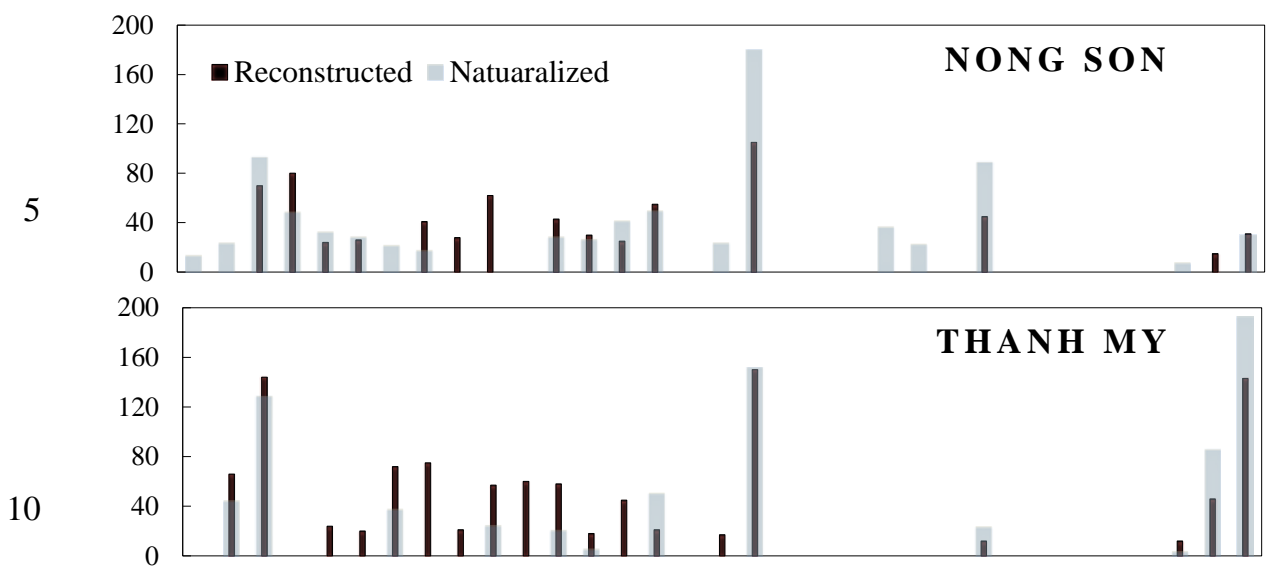

15
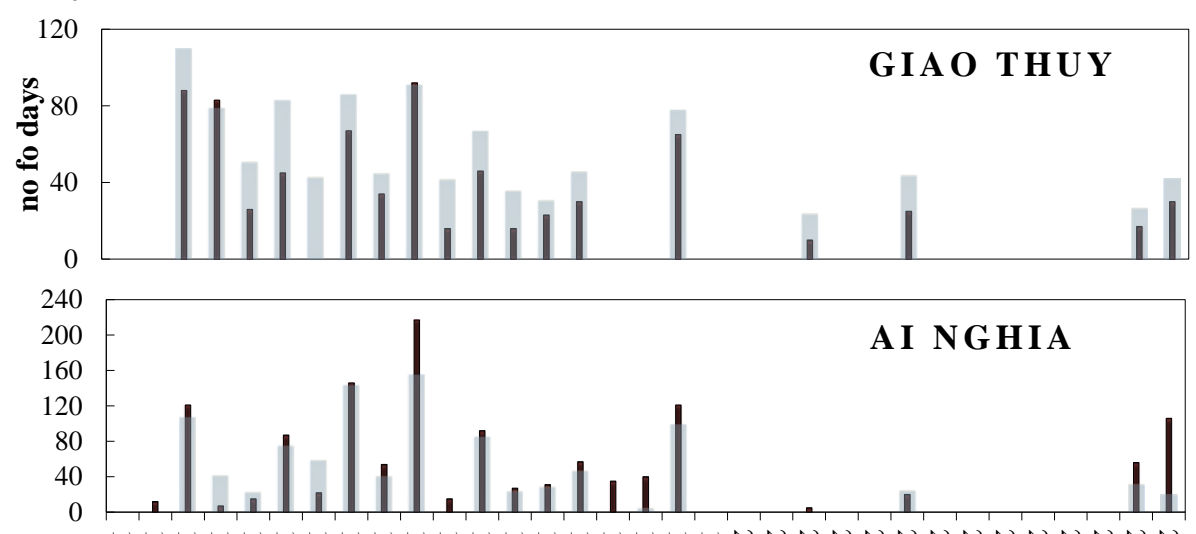

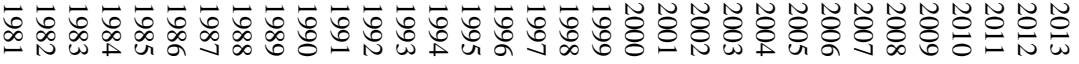

Year

Figure S6. Number of hydrological drought days at four stations for both naturalized and reconstructed condition. 


\section{Output uncertainty Plot}

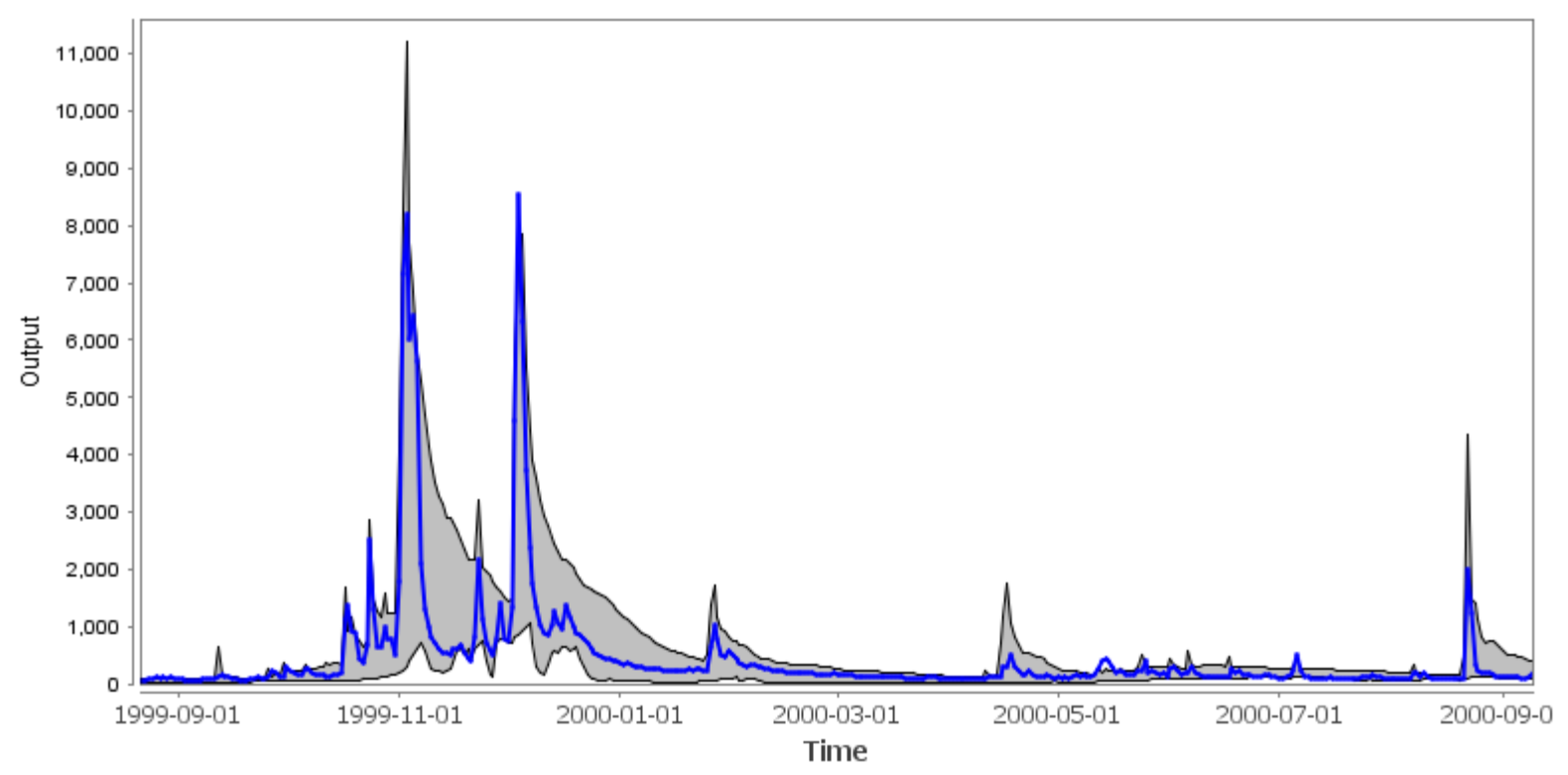

Figure S7. 5\% best simulations (range grey shaded) versus observed discharge (blue line) at Nong Son. 


\section{References}

MOIT: Decision Number 5538/QD-BCT, Decision on Song Con 1 and Song Con 2 Operation, Hanoi, 15.10.2008, Ministry of Investment and Trade (MOIT), Hanoi, Viet Nam, 2008.

MOIT: Decision Number 6801/QD-BCT, Decision on Dak Mi 4 Reservoir Operation,, Ministry of Investment and Trade, Socialist Republic of Vietnam, Hanoi, Viet Nam, 2011a.

10 MOIT: Decision Number 6801/QD-BCT, Decision on Dak Mi 4 Reservoir Operation, Hanoi, 23.12.2011, Ministry of Investment and Trade (MOIT), Hanoi, Viet Nam, 2011b.

MOIT: Decision Number 3711/QD-BCT, Decision on Song Bung 5 Reservoir Operation, Hanoi, 29.06.2012, Ministry of Investment and Trade (MOIT), 2012a.

MOIT: Decision Number 4537/QD-BCT, Decision on Song Bung 5 Reservoir Operation, Hanoi, 07.08.2012, Ministry of 15 Investment and Trade (MOIT), Hanoi, Viet Nam, 2012b.

MOIT: Ministry of Investment and Trade (MOIT), Decision Number 7878/QD-BCT, Decision on Song Tranh 2 Operation, Hanoi, 24.12.2012, Ministry of Investment and Trade (MOIT), Hanoi, Viet Nam, 2012c.

MOIT: Decision Number 2093/QD-BCT, Decision on Song Bung 4 Reservoir Operation, Hanoi, 04.04.2013, Ministry of Investment and Trade (MOIT), Hanoi, Viet Nam, 2013. 